\title{
Structuring Decision Problems \\ in Automated Control Systems \\ of Military Assignment
}

\author{
Vladimir V. Tolstykh* \\ Military Academy of the General Staff \\ of the Armed Forces of the Russian Federation \\ 100 Vernadsky, Moscow, 119571, Russia
}

Received 26.11.2016, received in revised form 20.12.2016, accepted 15.01.2017

In the interests of increase of efficiency of functioning of automated control systems for military purposes (ACS MP), the issue of formalizing the description of goals and means in the decision making (DM) problems determining the status of the control object, has been discussed, as it is necessary for the structuring and proper positing of these problems.

Keywords: automated control systems of military assignment, decision making,control object.

Citation: Tolstykh V.V. Structuring decision problems in automated control systems of military assignment, J. Sib. Fed. Univ. Eng. technol., 2017, 10(2), 271-276. DOI: 10.17516/1999-494X-2017-10-2-271-276.

\section{Структуризация задач принятия решений}

\author{
в автоматизированных системах \\ управления военного назначения
}

В.В. Толстых

Военная академия Генерального штаба Вооруженных сил Российской Федерачии Россия, 119571, Москва, пр. Вернадского, 100

В интересах повышения эффективности функционирования автоматизированных систем управления военного назначения (АСУ ВН) рассмотрен вопрос формализации описания целей и средств в задачах принятия решений (ПР) при определении состояния объекта управления, так как это необходимо для структуризации этих задач и правильной их постановки.

Ключевые слова: автоматизированные системы управления военного назначения, принятие решения, объект управления.

(C) Siberian Federal University. All rights reserved

* Corresponding author E-mail address: oficer.1978@mail.ru 
Для формирования оптимального воздействия на объект управления (под которым будем понимать силы и средства воздействия на противника) в АСУ ВН есть необходимость принятия решений (ПР) в условиях неполной исходной информации и быстроменяющейся обстановки. Задача ПР, возникающая при управлении силами и средствами воздействия на противника, является сложной и многокритериальной, при этом существуют достаточно развитые методы моделирования объектов управления, позволяющие выделять и решать элементарные задачи ПР, рассматриваемые в данной статье. Предполагается, что сложные процессы ПР могут быть составлены из элементарных $[1,2]$.

В литературе [3-5] задачи ПР представляются кортежами различной длины, определяемыми сложностью решаемой задачи, состоящими из классов свойств среды ПР и их взаимных отображений. В классической постановке [5] задача ПР имеет вид двойки $\{\Omega, O П\}$, содержащей множество альтернатив $(\Omega)$ и принцип оптимальности $(O П)$. Наличие множества альтернатив определяет представление цели в неявном виде, что приводит к задаче выбора лучшей альтернативы в соответствии с выбранным принципом оптимальности. Элементарной задачей ПР будем считать тройку $\{G, S, f\}$, где $G$ - цель решения задачи, $S$ - совокупность средств достижения цели и $f$ - правила пользования средствами достижения цели. Такое представление отражает содержательную постановку и словесное описание задачи. Описание цели должно содержать все требования, ограничения и другие условия, которые необходимо учесть в процессе решения. Очень важно иметь в описании полный перечень всех доступных средств $S$, так как наличие средств является основой для успешного достижения цели. Задача ПР возникает, когда некоторые элементы тройки не заданы или заданы не полностью [6]. Чаще всего решаются задачи ПР при неизвестных правилах выбора средств и пользования ими, поэтому основная часть работ по ПР посвящается выявлению, моделированию и построению этих правил при участии лица, принимающего решение (ЛПР). В интересах повышения эффективности функционирования АСУ ВН рассмотрен вопрос формализации описания целей и средств в задачах принятия решений (ПР) при определении состояния объекта управления, так как это необходимо для структуризации этих задач и правильной их постановки.

Примем, что в исходном положении у нас имеется содержательное словесное описание задачи ПР. Структуризацию будем считать законченной, когда цели и средства их достижения будут представлены в виде формализованных переменных и между ними будет установлено соответствие, показывающее, какие средства для каких целей могут или должны быть использованы.

Структуризацию задачи можно условно разделить на несколько этапов:

1) построение неструктурированной модели в пространстве свойств среды ПР;

2) анализ измеримости свойств среды ПР;

3) структуризация свойств, т.е. представление их в виде переменных среды ПР;

4) упорядочение значений свойств среды ПР.

На первом этапе строится неструктурированная модель задачи в пространстве свойств. Для этого проводится анализ содержательного описания задачи, в результате чего формируются множества используемых в задаче свойств целей и средств и строится соответствие $(\Gamma)$ между этими множествами:

$$
\Gamma: X \rightarrow G
$$


где $X$ - свойства средств; $G$ - свойства цели.

Для того чтобы была возможна формализация законов отображения $(\Gamma)$, свойства $X$ и $G$ должны быть представлены в измеримой форме.

Всякое свойство выражается множеством своих проявлений. Для анализа измеримости необходимо путем физического или мысленного эксперимента определить и перечислить все проявления рассматриваемого свойства, т.е. определить:

$$
X=\left\{x_{1}, x_{2}, \ldots, x_{i}, \ldots, x_{h}\right\} .
$$

Свойство альтернативно, если все его проявления попарно несовместимы. Такое свойство может быть представлено переменной, областью значений которой выступает множество альтернативных проявлений, т.е.

$$
X=\left\{x^{1}, x^{2}, \ldots, x^{i}, \ldots, x^{h}\right\} .
$$

В математике измеримость связывается с заданием на множестве проявлений неотрицательной (I), аддитивной (II) и монотонной (III) функции, а также (IV) - единицы измерения, являющейся, как и все математические объекты, идеализацией реально существующих мер в реальных системах [3]. Для описания реальных свойств среды ПР используем наиболее слабое понятие меры, для которой обязательно только условие (IV), что фактически соответствует требованию альтернативности проявлений. Следовательно, свойство измеримо, если его проявления альтернативны.

Множество проявлений свойств среды ПР будем считать полным, если свойство в принципе не может иметь других, кроме перечисленных или порождаемых по заданному закону, проявлений.

Альтернативность проявлений свидетельствует о сложности свойства. В этом случае представление в виде переменной $X$ реализуется классификацией совместимых проявлений и совместимость рассматривается совместимость как эквивалентность $(E)$, т.е.

$$
\begin{aligned}
& X=\left\{x^{1}, x^{2}, \ldots, x^{j}, \ldots, x^{t}\right\} ; \\
& x^{j}=\left\{x_{1}{ }^{j}, x_{2}{ }^{j}, \ldots, x_{S_{j}}{ }^{j}, \ldots, x_{\Gamma_{j}}{ }^{j}\right\}, \\
& x_{S_{j}}{ }^{j} E x_{1}{ }^{j} ; S_{j}=\overline{1, \Gamma_{j}} ; \overline{j=1, t} ; \Sigma \Gamma_{j}=h .
\end{aligned}
$$

Как видим, сложное свойство имеет сложные значения, представленные классами совместимых проявлений. Отношение совместимости - наиболее тонкая эквивалентностью на множестве проявлений. Исходя из условий решаемой задачи может быть задана более грубая эквивалентность, крайним случаем которой выступает эквивалентность всех проявлений. Тогда свойство выражается двухальтернативной переменной:

$$
\begin{aligned}
& X=\left\{x^{0}, x^{1}\right\} ; \\
& x^{0}=\varnothing, x^{1}=\left\{x_{1}, x_{2}, \ldots, x_{i}, \ldots, x_{h}\right\} ; \\
& x_{i} E x_{1} ; i=\overline{1, h} .
\end{aligned}
$$


Совместимость указывает на то, что проявления - это значения разных составляющих $\mathrm{x}_{\mathrm{i}}$ сложного свойства $X$. Составляющие могут быть установлены углубленным анализом содержательного описания данного свойства (в аспекте решаемой задачи) и его разложением:

$$
X=\left\{X_{1}, X_{2}, \ldots, X_{i}, . . X_{k}\right\} .
$$

Структуризация свойства $X$ осуществляется путем разложения его на измеримые составляющие и агрегирования этих составляющих (если необходимо) обратно в одну переменную $X$, так как в выражении (6) составляющие $X_{i}$ могут оказаться измеримыми в различных шкалах, то единственно возможным способом агрегирования служит декартово произведение:

$$
X=\left\{X_{1} * X_{2} * \ldots * X_{i} * \ldots * X_{k}\right\}
$$

Если составляющие в (6) представлены областями значений:

$$
X=\left\{X_{1} * X_{2} * \ldots * X_{i} * \ldots * X_{k}\right\},
$$

то переменная $X$ будет иметь область значений:

$$
\begin{aligned}
& X=\left\{x^{1}, x^{2}, \ldots, x^{j}, . ., x^{n}\right\} \\
& x^{j}=\left\{x_{1}^{j_{1}}, x_{2}{ }^{j_{2}}, \ldots, x_{i}^{j_{i}}, \ldots, x_{k}^{j_{k}}\right\} \\
& |X|=\prod n_{i}=n
\end{aligned}
$$

Отношение порядка $R_{i}=>$ определяет в декартовом произведении частичный порядок на множестве агрегированных значений $\left\{x^{j}\right\}$. Если условия задачи допускают факторизацию этих значений, то их число сокращается до величины

$$
|X|=\sum_{i}-(k-1) .
$$

Соотношения (9) и (10) справедливы только при невзаимодействующих составляющих $X_{i}$. Взаимодействие свойств определяется существованием отношения между свойствами или их значениями.

Взаимодействие значений свойств выражается в том, что принятие каких-либо значений одним или несколькими свойствами ограничивает в декартовом произведении области возможных значений других составляющих свойств. Причиной этого служит несовместимость составляющих значений $x_{i}^{j i}$ в наборах (9). Такие наборы $x^{j}$ невозможны и исключаются из области значений $X$.

Взаимодействие составляющих может быть установлено при условии их сравнимости и определяется как взаимная неравноценность $X_{i}$. Если на $\left\{X_{i}\right\}$ задан полный строгий порядок:

$$
\mathrm{R}_{\mathrm{x}} \Rightarrow \mathrm{X}_{1},>\mathrm{X}_{2}>\ldots>\mathrm{X}_{\mathrm{i}}>\ldots>\mathrm{X}_{\mathrm{k}}
$$

это значит, что на множестве порядков $R_{i}$ существует отношение включения:

$$
\begin{array}{r}
R_{1} \supset R_{2} \supset \ldots \supset R_{i} \supset \ldots \supset R_{k}, \\
-274-
\end{array}
$$


определяющее, что $R_{1}$ грубее $R_{2}$ и т.д. Упорядочение составляющих делает более тонким по сравнению с (10) порядок структуризации переменной $X$. В предельном случае отношение включения (I2) превращается в отношение принадлежности:

$$
R_{k} \in R_{k-1} \in \ldots \in R_{i} \in \ldots \in R 1 .
$$

При этом на множестве составляющих $X_{i}$ устанавливается лексикографический порядок, что приводит к полному строгому упорядочению декартова произведения (9):

$$
x^{1} \succ x^{2} \succ \ldots \succ x^{j} \succ \ldots \succ x^{n} .
$$

Предложенный общий подход - основа структуризации описания целей и средств. Однако степень сложности и изученности свойств, их роль в решаемой задаче и другие факторы порождают ряд особенностей структуризации.

Структуризация средств и выражение их свойств в виде одной переменной приводит к построению допустимого множества альтернатив $X$, вопросы дальнейшего сокращения этого множества с использованием доступной информации о правилах выбора рассматриваются в [5].

Согласно положениям теории принятия решений [6], измерителем цели является критерий. Если цели измеримы, то они и есть прямые критерии в задаче ПР. Структуризация множества целевых свойств $\left\{G_{i}\right\}$ по выражениям (6) - (14) приводит к построению прямых критериев задачи ПР. Более того, этим же методом может быть построен и упорядочен глобальный критерий $G$. При этом за счет анализа взаимодействий частных критериев и исключения невозможных наборов их значений сокращается объем критериального пространства.

Упорядочение критериев обладает одной важной особенностью. В задаче ПР средства отображаются на цели, поэтому необходимо их комплексное рассмотрение с учетом всей информации об отображении, имеющейся в содержательном описании задачи. При этом отображение может быть представлено как измерение средств (измеряемого множества) с помощью целей (эталонного множества), а решение задачи - как построение шкалы:

$$
-: X \rightarrow G
$$

В этих условиях отношения, существующие на $X$, отображаются в критериальное пространство $G$ и влияют на упорядочение критериев. Это объясняется тем, что трудность достижения того или иного критериального значения (цели) определяется необходимым для этого объемом средств и интенсивностью их использования. Для того чтобы между двумя значениями $g^{l}$ и $g^{2}$ критерия $G$ существовало индуцированное отношение порядка $R_{X G}=>$, необходимо, чтобы между множествами связанных с ними свойств $X_{g}{ }^{l}$ и $X_{g}{ }^{2}$ соответствующими сужениями $\Gamma_{x g}{ }^{l}$ и $\Gamma_{x g}{ }^{2}$ графика соответствия (1) существовало отношение включения:

$$
X_{g^{l}} \supseteq X_{g^{2}} \wedge \Gamma_{x g^{l}} \supseteq \Gamma_{x g^{2}} \wedge \exists X_{i} \in X_{g^{2}} \wedge \Gamma_{x_{i} g^{l}} \supset \Gamma_{x_{i} g^{2}} \Rightarrow g^{l} \succ g^{2} .
$$

Отношение $R_{X G}$ доминирует над естественным порядком критерия в случае их несовпадения.

После структуризации управляющих воздействий получается множество управляющих переменных (формализованные документы (приказы) боевой готовности), которые и есть 
основные критерии классификации состояний. Особенность задач управления состоит в том, что критерии и состояние, как правило, описываются дискретными неметрическими переменными. Это объясняется тем, что переход из одного класса состояний в другой сопровождается качественными изменениями (или угрозой таковых) и является для объекта уникальным событием, в то время как математическая мера применима исключительно для массовых событий. В этих условиях правило принятия решения - представляется в виде дискретного соответствия.

Рассмотренные в работе этапы структуризации - неформализованные творческие процедуры. Они могут рассматриваться как экспертные алгоритмы, реализующие предложенный подход.

\section{Список литературы}

[1] Осис Я.Я. Автоматика и вычислительная техника. Журнал Латвийского университета, 1969, 6, 44-50. [Osis Ya.Ya. Automatics and computer facilities. Magazine of the Latvian university, 2069, 6, 44-50 (in Russian)].

[2] Новожилова Н.В., Маркович 3.П. Обработка топологической граф-модели объекта и выделение диагностического оптимального множества параметров (ДОМПТОГРАМ). ГосФАП - П006789. М.: Гос. фонд алгоритмов и программ, 1983. 150 с. [Novozhilova N.V., Markovich Z.P. Processing topological the count-models of object and allocation of diagnostic optimum set of parametres (DOMPTOGRAM). GosFAP - P006789. Moscow, Gos. fond algoritmov i programm, 1983, 150 p. (in Russian)].

[3] Озерной В.М. Труды Института проблем управления. М., 1974,. 5, 3-15 [Ozernoy V.M. Works of Institute of problems of management. Moscow, 1974, 5. 3-15 (in Russian)].

[4] Борисов А.Н., Алексеев А.В., Крумберг О.А. и др. Модели принятия решений на основе лингвистической переменной. Рига: Зинатне, 1982. 256 с. [Borisov A.N., Alekseev A.V., Krumberg O.A. et.al.. Models of decision-making on the basis of a linguistic variable. Riga, Zinatne, 1982, 256 p. (in Russian)]. 